\section{Sipat-suri sa piling akda ni Pedro S. Dandan: Implikasyon sa pagtuturo ng maikling kwentong Filipino sa antas tersarya}

\author{
Noval, Arnel $\measuredangle$ \\ Cebu Technological University - Main Campus, Philippines (arnelnoval663@gmail.com)
}

Received: 9 December 2020

Available Online: 5 March 2021

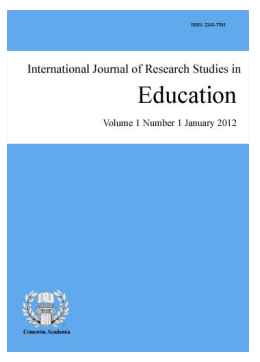

ISSN: 2243-7703 Online ISSN: 2243-7711

OPEN ACCESS

\title{
Abstract
}

The students' weakness in analyzing and understanding literary texts, and the declining interest in reading Filipino literature motivated the researcher to conduct this study. The study aims to analyze the selected works of P. S. Dandan and use it as basis to formulate implications in teaching Filipino short stories in the tertiary level. Specifically, it analyzed the plot structure of the text according to introduction, body/middle and end and the social issues which can be reflected in the literary texts. The study utilized a qualitative method of research in the form of content analysis. The results revealed that poverty is the most prominent social issue in the texts which caused chaotic problems such as issues on health and other abuses in the society. From these findings, it was concluded that through social issues reflected in the plot structure of a short story one can further shape the artistry of one's work.

Keywords: sipat-suri; pagtuturo; maikling kwento; Filipino; tersarya 


\section{Sipat-suri sa piling akda ni Pedro S. Dandan: Implikasyon sa pagtuturo ng maikling kwentong Filipino sa antas tersarya}

\section{Panimula}

Ang kahalagahan ng panitikan sa sandaigdigan at sa bawat nilalang ay kawangis nang walang katapusang pag-agos ng tubig sa batis. Magwawakas lamang ito kung mabubura at mawawala sa kamalayan ang mga titik sa sanlibutan at kung mawawalan ng talino at bisa ang tao sa pagpapahayag ng damdamin, kaisipan at karanasan (Sauco, Papa, at Geronimo, 2004). Isang halimbawa ng akdang pampanitikan na kakikitaan at binuhusan ng husay sa pagpapahayag ay ang maikling kwento. Ngunit, ang ganitong obra ay nanganganib na hindi mapansin at mapasawalang-bahala na lamang dahil sa tila ba nagiging dayuhan sa sariling panitikan ang ating mga kabataan. Sa patuloy at mabilis na pagbabago, dumaraming libangang dulot ng makabagong teknolohiya at impluwensyang banyaga, waring unti-unting bumababaw ang interes ng mga mag-aaral sa panitikang Filipino.

Ito ang kahinaang naobserbahan ng mananaliksik sa kanyang mga mag-aaral sa pagtuturo ng asignaturang Filipino at Panitikan. Tila ba nagiging dayuhan sa sariling panitikan ang mga mag-aaral. Patunay nito ang kahinaan ng mga mag-aaral sa pag-unawa ng mga akdang pampanitikan. Dagdag pa ang mali-maling gramatika sa pagsulat ng pangungusap at mga talata, higit lalo ang balu-baluktot na pagsasalita ng wikang Filipino. Ang mga kahinaang ito ang nagtulak sa mananaliksik upang isagawa ang pag-aaral.

\subsection{Ang mga nagwaging obra ni Pedro S. Dandan sa Gawad Palanca}

Si Pedro S. Dandan ay isang manunulat na nagtamo ng prestihiyosong gawad sa Don Carlos Palanca Memorial Awards sa kanyang angking husay sa pagsulat ng maikling kwento. Ang kanyang mga obra ang magsisilbing daan upang maisakatuparan ang pag-aaral na ito sa kadahilanang may malaki itong ambag sa pagpapalaganap at kalinangan ng Panitikang Filipino. Iginawad sa akdang "Mabangis na Kamay... Maamong Kamay" ang ikalawang gantimpala sa Timpalak-Palanca noong 1950-1951 sa kategorya ng maikling kwento. Nagwagi naman ng ikatlong gantimpala sa kaparehong patimpalak ang kanyang akdang "Lakas" sa taong 1957-1958. Dagdag pa nito, ang akdang “Anino ng Kanyang Ama” ang nakakuha ng ikalawang pwsesto sa taong 1965-1966 sa kaparehong kategorya.

\subsection{Realismo: Isyung panlipunang masasalamin sa maikling kwentong Filipino}

Sa pagtitiyak na magabayan ang pagsusuri, iniangkla ang pag-aaral sa mga isyung panlipunang mahihinuha sa mga akda sa realismong pananaw. Ipinaglalaban ng pananaw realismo ang katotohanan kaysa kagandahan. Sa anomang tao, bagay at lipunan, para sa mga realista ay dapat maging makatotohanan ang paglalahad nito. Higit nilang kinikilingan ang uri ng paksa sa isang akda. Kung kaya, hindi kataka-taka kung ang magiging paksa nito ay nakapokus sa sosyo-politikal, kalayaan at katarungan para sa naaapi (Villafuerte at Bernales 2008).

Pinagsusumikapan ng mananaliksik na mapatunayan na ang akda ni P.S. Dandan ay sumasalamin sa mga isyung panlipunang nararanasan ng pangkalahatan. Pinaiiral ng realismong pananaw ang mga usaping nararanasan ng mga mamamayan gaya ng pagkagutom, kahirapan, mababang kalidad ng edukasyon, laganap na korapsyon at iba pang sakit ng lipunan.

\subsection{Pagsusuri sa maikling kwentong Filipino bilang salamin ng lipunang Pilipino}

Ang maikling kwento ay isang mabisang kasangkapan upang maipakikilala ang pagkakakilanlan ng isang lahi at ang kanyang natatanging kakayahan. Sa tulong ng maikling kwento, naipamamalas ang katotohanan ng buhay, ang kanais-nais at hindi kanais-nais na mga pangyayari sa mga mambabasa. Nailalahad din nito kung 
Sipat-suri sa piling akda ni Pedro S. Dandan: Implikasyon sa pagtuturo ng kwentong Filipino sa antas tersarya paano nalutas ng pangunahing tauhan ang pagsubok gamit ang talino at tibay ng loob (Villafuerte, 2000). Sa madaling salita, ang maikling kwento ay isang matibay at mabisang kasangkapan sa pagmumulat sa kamalayan ng mga mag-aaral sa mga pangyayari sa kapaligiran at makisangkot sa pagpapayaman ng panitikang Filipino.

Binanggit nga nina Lalic at Matic (2004), ang tunay na panitikan ay ang tapat na paghahabi o paglilok gamit ang mga panitik upang isalarawan ang tunay na buhay at karanasan. Ito ay pinagsunod-sunod ayon sa pansariling karanasan ng tao batay sa ninanais na paraan ng pagpapahayag. Karanasang sumasalamin sa pagkatao ng isang nilalang at tagapaghubog ng pagkatao sa mga sumusunod na henerasyon.

Maipamamalas lamang ang kabutihang hatid ng maikling kwento kung ang sinomang mambabasa ay may sapat na kaalaman sa panunuring pampanitikan. Ang panunuring pampanitikan ay hindi lamang sumusuri o nagpapakahulugan sa mga pangyayari sa sanlibutan kundi ito ay isang paraan ng paghihimay sa pagkakakilanlan ng isang tao, ang kanyang anyo, ugali, gawi, pananalita at maging sa pakikisalamuha sa kapwa at kinabibilangang lipunan (Villafuerte, 2000). Ganito rin ang paniniwala ni Dinglasan (halaw kay Oroc, 2012), na ang sukatan ng akdang pampanitikan ay sa pamamagitan ng paghihimay o pagsusuri sa kabuoan at nilalaman nito. Maaaring sa paglalahad, pag-aanalisa, pagkikilatis at maaaring sa pag-aaral, pag-iiksamen o pagsisiyasat sa elementong bumubuo sa akda. Wala itong pagkiling, makatotohanan at nagbibigay ng katwiran batay sa mga pagpapatunay.

\subsection{Layunin ng pag-aaral}

Nilayon ng pag-aaral na ito na masuri ang piling akda ni P.S. Dandan. Tinitiyak din nito na matugunan ang mga sumusunod na mga katanungan: (1) Ano-ano ang mga elemento ng piling akda sa banghay batay sa panimula, gitna at wakas? (2) Anong isyung panlipunan ang masisilayan sa banghay ng kwento? (3) Batay sa mga natuklasan, anong implikasyon sa pagtuturo ng maikling kwentong Filipino sa antas tersarya ang mabubuo?

\section{Metodolohiya}

\subsection{Disenyo ng pag-aaral}

Ang disenyong ginamit ng pag-aaral ay kwalitatibong pananaliksik sa anyong kontent analisis na inasagawa sa pamamagitan ng masusing pagsisiyasat at paghihimay sa nilalaman ng piling akda ni P.S. Dandan. Hinimay ang elemento ng mga piling akda ayon sa banghay gaya ng panimula, gitna at wakas. Pinagtuonan din ng pag-aaral ang paghihimay sa realismong pananaw na masisilayan sa banghay ng kwento upang makabuo ng implikasyon sa pagtuturo ng maikling kwentong Filipino.

Matapos matiyak na sapat na ang mga kinakailangang sanggunian, sinimulan ang paghihimay sa elemento o sangkap ng maikling kwento ayon sa banghay batay sa panimula, gitna at wakas upang mas mapadali ang pagsusuri sa sintaktikal na estruktura ng mga akda. Sinundan naman ito ng paglikom ng mga datos sa pamamagitan ng paghimay sa realismong pananaw na masisilayan sa mga akda na inihahantad sa estrukturang sintaktikal ng banghay ayon sa panimula, gitna at wakas.

Sa pagtitiyak sa validity at reliability ng mga kinalabasan ng pag-aaral, sinangguni ng mananaliksik ang limang panel na dalubhsa sa larangan ng panitikan at pananaliksik. Isinaalang-alang ng mananaliksik ang mga suhestiyon at rekomendasyon ng panel upang matiyak na ang mga resulta ay naangkop at naaayon sa pagtamo ng mga nilalayon ng pag-aaral.

\subsection{Pinanggalingan ng mga datos}

Ang mga akda ni Pedro S. Dandan na "Mabangis na Kamay... Maamong Kamay”, "Lakas" at "Anino ng Kanyang Ama" ang napili at pinaghanguan ng mga datos sa isinagawang pagsusuri. Ito ay masusing binasa at hinimay upang ang mga datos na nalikom ay magamit sa pag-aaral. Sa pagtiyak sa kawastuhan ng mga datos 
Noval, A.

sinangguni rin ang internet, mga batayang aklat, tesis at disertasyon.

\subsection{Limitasyon ng pag-aaral}

Nakasentro ang pag-aaral na ito sa pagsusuri sa banghay ng maikling kwento ayon sa panimula, gitna at wakas. Hinimay rin ang mga isyung panlipunang mamamalas sa akda upang makabuo ng mga implikasyon sa pagtuturo ng maikling kwentong Filiino sa antas tersarya.

\section{Resulta at pagtatalakay}

Inilalahad sa bahaging ito ang pagsusuri sa banghay ng piling akda ni P.S. Dandan ayon sa simula, gitna at wakas. Kaugnay nito, matutunghayan din dito ang isinigawang pagsusuri sa mga isyung panlipunang masasalamin sa mga akdang ito.

\subsection{Mabangis na kamay... maamong kamay}

Ang akdang "Mabangis na Kamay... Maamong Kamay" ay kwento ng isang kampyong boksingero na si Battling Kulas na dahil sa pagnanais na makahanap ng lunas sa maysakit na anak at dala ng matinding pagsisi ay huminto sa pagboboksing at tinalikuran ang kinang ng entablado sa paniniwalang ito ay kasalanan sa mata ng Diyos. Naniniwala rin si Battling Kulas na ang sakit na dumapo sa pinakamamahal na anak ay parusa sa kanya ng Panginoon.

Mapapansin sa panimulang bahagi ng banghay na kakikitaan ito ng iba at ibang parirala tulad ng pariralang pandiwa na naghahakot ng basura, pariralang pang-uri na nangingitim na pawis at pariralang pang-abay na isang malaking tiklis ng basura.

Pinalulutang ng panimulang banghay ang kahirapan. Ang mga pariralang naghahakot ng basura, nangingitim na pawis at isang malaking tiklis ng basura ay pawang nagpapahiwatig ng kahirapan. Lantaran ang larawan ng kahirapang dinaranas ng pangunahing tauhan sa kwento. Ang naging kabayaran ng pagtalikod ni Battling Kulas sa pagboboksing ay paghihikahos at kasalatan sa buhay. Dahil dito, wala silang ibang makapitan kung di ang mangalakal ng basura upang matustusan ang kanilang pangangailangan. Ang ganitong pagsasalarawan, nakapanlulumo man ay laganap at patuloy na kinakaharap ng ating bansa. Ayon sa sarbey ng Social Weather Station (SWS), mahigit 10.5 Milyong pamilyang Pilipino ang nagpapabilang sa mahirap. Dagdag pa nito, Sa unang quarter ng 2012 tinatayang tumaas ng $23.8 \%$ o tinatayang 4.8 milyong pamilya ang nakaranas ng pagkagutom (Gavilan, 2016; Balita, 2016).

\section{Talahanayan 1}

Realismong pananaw sa akdang "Mabangis na Kamay... Maamong Kamay"

\begin{tabular}{|c|c|}
\hline Mabangis na Kamay... Maamong Kamay & Realismong Pananaw \\
\hline $\begin{array}{ll}\text { Panimula } \\
-\quad \text { naghahakot ng basura } \\
-\quad \text { nangingitim na pawis } \\
\text { - } \quad \text { isang malaking tiklis ng basura }\end{array}$ & $\begin{array}{l}\text { Ang kahirapan ay nangununang sakit na } \\
\text { nagpapasakit sa tao at sa lipunan. }\end{array}$ \\
\hline $\begin{array}{l}\text { Gitna } \\
-\quad \text { inihahatid na patay } \\
\text { - bunga ng tila ibig kumalat na salot } \\
\text { inaapoy ng lagnat }\end{array}$ & $\begin{array}{l}\text { Ang suliraning pangkalusugan ay isa sa mga } \\
\text { resulta ng kahirapang laganap sa isang lipunan. }\end{array}$ \\
\hline $\begin{array}{l}\text { Wakas } \\
-\quad \text { napaluhod siya } \\
-\quad \text { isang malakas na dalangin }\end{array}$ & $\begin{array}{l}\text { Sa panahon ng pangangailangan nasusukat ang } \\
\text { tibay ng pananalig ng isang nilalang. }\end{array}$ \\
\hline
\end{tabular}

Sa gitnang bahagi ng banghay ng kwento tulad ng mga pariralang bunga ng tila ibig kumalat na salot, inihahatid na patay at inaapoy ng lagnat ay kasasalaminan ng suliraning pangkalusugang kinakaharap ng mga tauhan. Ang pagkalat ng epidemya na nagdudulot sa pagkakamatay ng mga tao sa kanilang bayan ng hindi man

40 Consortia Academia Publishing (A partner of Network of Professional Researchers and Educators) 
Sipat-suri sa piling akda ni Pedro S. Dandan: Implikasyon sa pagtuturo ng kwentong Filipino sa antas tersarya lang naipapakonsulta o natitingnan ng doktor. Ang ganitong sitwasyon ay may kaugnayan sa kasalukuyang panahon sapagkat marami sa mga mahihirap ay namamatay dulot ng sakit na wala man lang kalaban-laban. Kung makapunta man sa ospital at makapagpagamot kulang naman sa pasilidad at equipment kaya hindi nakapagbibigay ng karampatang lunas. Ayon kay Dr. Reyte, "higit na mahirap ang kalagayan ng mahihirap sa malalayong pook na apektado ng mga trahedya tulad ng naganap na pananalanta ng bagyong Yolanda. Naroon pa rin ang kakulangan ng mga manggagamot at pasilidad sa mga malalayong pook na kailangang daluhan ng pamahalaan" (CRI Online Filipino, 2015).

Malinaw na ipinalulutang ng pangwakas na banghay ang kaugalian at kultura ng mga Pilipino sa taimtim na pananalig sa Diyos tulad ng mga pariralang napaluhod siya at isang mataos na dalangin. Ang mga pariralang ito ay lantarang nagpapahiwatig ng malakas na pananalig ng pangunahing tauhan sa Panginoon sa kabila ng nakalulunos na pangyayaring naganap sa kanyang buhay. Nakalulungkot ang naging kapalaran ng pangunahing tauhan na si Battling Kulas sapagkat nabawian ng buhay ang kanyang anak, Arya Ito, sa kanya mismong mga kamay. Sa gitna ng matinding delubyo at kalungkutan ang Panginoon ang siyang sandigan. Ang pag-uugaling taglay ni Battling Kulas ay sumasalamin sa nakararaming Pilipino na namamanata at may malakas na pananalig sa nakatataas. Ang pagiging relihiyoso ng mga Pilipino maging ito ay ipinapahayag sa Panginoon, kaugnay sa mga espiritu at mga supernatural o di kaya ay sa mga anito ay kilala sa buong mundo (Philippine Social Science Council, 2003).

\subsection{Lakas}

Ang akdang "Lakas" ay tungkol sa matagumpay na buhay ni Ingkong Simo sa larangan ng palakasan na bagamat humarap sa matitinding dagok o pagsubok at kahirapan na naging sanhi ng kanyang pagiging paralitiko ngunit hindi nagpatinag sa hamon ng buhay at muling bumangon at kinilala sa kanyang angking karunungan.

Inihahantad sa panimulang bahagi ng banghay ang pariralang ang namimitig na mga binti at bago siya maging paralitiko ang eksena kung saan tinitiis na lamang ni Ingkong Simo ang kanyang pagkaparalisa nang hindi man lang natitingnan ng doktor habang inaalala ang dating kabataan noong siya ay malakas pa. Ang ganitong eksena ay pasakit sa maraming Pilipino na dulot ng kakapusan ay wari bang papasok sa butas ng karayom ang pagkuha ng kalidad na serbisyong medikal.

\section{Talahanayan 2}

Realismong pananaw sa akdang "Lakas”

\begin{tabular}{|c|c|}
\hline Lakas & Realismong Pananaw \\
\hline $\begin{array}{l}\text { Panimula } \\
-\quad \text { ang namimitig na mga binti } \\
\text { bago siya naging paralitiko }\end{array}$ & $\begin{array}{l}\text { Karapatan ng bawat isa ang mabigyan ng } \\
\text { kalidad na serbisyong pangkalusugan ngunit } \\
\text { marami pa rin ang nagkakasakit na hindi man } \\
\text { lang nabibigyan ng karampatang lunas }\end{array}$ \\
\hline $\begin{array}{l}\text { Gitna } \\
-\quad \text { pinipilit na maiahon sa paso ang kariton } \\
\text { iginupo siya ng biglang pagtakas ng kanyang lakas }\end{array}$ & $\begin{array}{l}\text { Ang kahirapan ay hindi sagwil sa pagnanais ng } \\
\text { tao na makamit ang mithiin sa buhay }\end{array}$ \\
\hline $\begin{array}{l}\text { Wakas } \\
-\quad \text { labis na pagkagahaman ni Lupo }\end{array}$ & $\begin{array}{l}\text { Marami sa kabataan na sa murang edad ay } \\
\text { nakararanas ng panlalamang sa kanilang kapwa } \\
\text { bata }\end{array}$ \\
\hline
\end{tabular}

Ayon kay Romualdez et al. (2011), laganap ang nakahahawang sakit at nangangailangan ng karampatang atensyon. Mahalagang matugunan ng gobyerno ang ganitong usapin upang maibsan ang pagpapakasakit at ang pagtitiis ng nakararaming Pilipino.

Kapansin-pansin sa gitnang bahagi ng akda ang paghahantad sa pagsusumikap ng pangunahing tauhan sa kabila ng kahirapan at mga pagsubok na kanilang dinaranas. Kahanga-hanga ang pagsusumikap ni Ingkong Simo sa pagtatrabaho sa paghahakot ng kanilang mga produkto upang matugunan ang pangangailangan ng kanyang pamilya. Ang kalagayang ito ay repleksyon ng di matatawarang pagsusumikap ng mga Pilipino upang madaig 
Noval, A.

ang mga pagsubok sa buhay at manatiling matatag sa gitna ng kahirapan.

Ang kahirapan ang ugat ng sanga-sangang problema na kinakaharap ng nakararaming Pilipino. Ang nakalulungkot pa ay ang katotohanang ang mga nagpapakahirap sa pagtatanim, pagsasaka at pangingisda ang nangungunang sektor ng pinakamahirap sa ating bansan (Philippine Statistics Office, 2014).

Ayon sa sarbey ng Social Weather Station (SWS), mahigit 10.5 Milyong pamilyang Pilipino ang nagpapabilang sa mahirap. Sinupurtahan naman ito ng ulat ng Philippine Statistics Authority (PSA), na ang mga mangingisda, mga magsasaka at kabataan ang pinakamahihirap na sektor ng bansa. Ang mga datos na ito ay patunay lamang ng malalang sakit ng bayan. Sakit na pumipinsala sa mamamayan at sa lipunan kaya marapat lamang itong masolusyonan ng pangmatagalan (Philippine Statistics Office, 2014).

Masisilayan sa bahaging wakas ng banghay sa kwento na ang pariralang labis na pagkagahaman ni Lupo na kasasalaminan ng panunuya ng isang bata sa kapwa bata. Ang ganitong eksena ay kalimitang masasaksihan sa mga lansangan at sa mga paaralan. Ang pagmamaton o pambubully ay isang uri ng pananakit na pisikal o berbal na maaaring makaapekto sa mental at emosyonal ng biktima. Ayon sa Anti-Bullying Act of 2013, ang lahat ng paaralan sa elementarya at sekondarya ay kailangang gumawa ng mga polisiya upang ipagbawal ang pagmamaton at matulungan ang mga biktima nito (Perol, 2014).

\subsection{Anino ng kanyang ama}

Sa akdang "Anino ng Kanyang Ama" inilalahad ang pagnanais ni Merto na makapagsarili at buuin ang kinabukasan ng kanyang sariling pamilya ay pilit paring hinahadlangan ng kanyang ama na labis ang paniniwala sa Panginoon at pamamanata. Hanggang sa unti-unti niyang naramdaman ang anino ng kanyang ama na isinusuot sa kanyang hindi makatutol na anino ang lubid.

Inihahantad sa panimulang bahagi ng banghay ang pariralang sa santaon ang labinlimang kaban na nagpapahiwatig ng hindi pantay na paghahati-hati ng kita ng saka na pinaghirapan ni Merto. Inaasahan niya na ang ibibigay ng kanyang ama ay apatnapung kaban ng palay na sa taya niya ay tatagal ng dalawang buwan ang isang kaban ngunit kabalintunaan lamang ito sapagkat ang ibinahagi sa kanya ng kanyang ama ay labinlimang kaban lamang na pagkakasiyahin pa nila sa loob ng isang taon na kung iisipin ay kulang na kulang para sa lumalaki niyang pamilya. Ang eksenang ito ay hindi maitatago sa kasalukuyang lipunan sapagkat marami pa rin ang mapang-abuso at mapanlamang sa kapwa.

Inihahantad ng gitnang banghay, na litaw na litaw ang pagiging relihiyoso ng mga Pilipino na bahagi na ng buhay at nakaugat na sa kultura. Kilala ang mga Pilipino sa pagiging relihiyoso. Pinagdiriwang at ginugunita ng mga Pilipino ang kabanalan ng tagapaglikha sa abot ng makakaya. Ngunit minsan ang pagiging relihiyoso ay sobra na at minsan ay umaabot na sa pagkakataong kumukontrol o nananakot gamit ang kabanalan ng Panginoon tulad ng sa nangyari sa akdang “Anino ng Kanyang Ama.”

\section{Talahanayan 3}

Realismong pananaw sa akdang “Anino ng Kanyang Ama”

\begin{tabular}{ll}
\hline \multicolumn{1}{c}{ Lakas } & \multicolumn{1}{c}{ Realismong Pananaw } \\
\hline Panimula & Hindi pantay na pagbabahagi o panlalamang sa \\
mga magsasaka & \\
Gitna sa santaon ang labinlimang kaban & Mabisang panghuhugutan ng lakas ang \\
pananalig sa Diyos sa mga panahong \\
pinanghihinaan ng loob \\
inalihan siya ng takot sa Diyos sa bigat ng parusang \\
Kakas & Kahirapan ang ugat ng sanga-sangang suliranin \\
ng lipunan kabilang na ang pagkagutom at \\
kakulangang pangkalusugan
\end{tabular}

Sa loob ng pitong taon 1991-1998, ang mga Pilipino ay nagtataglay ng malakas na paniniwala at patuloy sa 
Sipat-suri sa piling akda ni Pedro S. Dandan: Implikasyon sa pagtuturo ng kwentong Filipino sa antas tersarya taimtim na pananalig sa Panginoon. Karamihan sa mga Pilipino ang naniniwala na nakapagbabago ang maliliit na mga gawa hinuhulma ng tao ang kanyang naging kapalaran at binibigyan niya ng kabuluhan ang sariling buhay (Abad, 2001).

Litaw na litaw sa wakas na bahagi ng banghay ang kahirapan ng kalagayan ng pangunahing tauhan. Ang boses ng kanyang asawa at anak sa kanyang isipan ay umaalingawngaw at nanunumbat sa kanyang kapabayaan at kakulangang masupurtahan ang pangangailangan ng pamilya. Sa isang sarbey na isinagawa ng SWS noong Disyembre 5-8 sa 1,200 na respondente sa fourth quarter ng 2015, 50 porsyento na katumbas ng 11.2 milyong pamilya ang nagsabing sila ay mahirap sa nakalipas na tatlong buwan. Ang karapatan sa pagkain ay kapantay ng karapatang mabuhay. Ito ay batayang karapatan ng lahat ng tao lalo na ang mga bata. Ang kagutuman at malnutrisyon ay mga problemang bunsod ng kawalan ng pambansang industriyalisasyon, ng tunay na repormang agraryo, ng trabaho at nakabubuhay na sahod ng manggagawa (Akap Bata Paty-List on Research and Education, 2012).

\subsection{Implikasyon sa pagtuturo ng maikling kwentong Filipino sa tersarya}

Kaakibat ng mabilis na pagbabago ay ang modernong teknolohiya at iba pang impluwensyang banyaga na naging sanhi sa nakapanlulumong pagbaba ng kawilihan ng mga mag-aaral sa pagkatuto ng sariling wika at pagtangkilik ng sariling panitikan. Isa sa mga indikasyon ng pagbaba ng kawilihan ng mga mag-aaral ay ang kahinaan sa pag-unawa at pagsusuri sa mga akdang pampanitikan dagdag pa ang mali-maling balarila sa pagsasalita o pagsusulat.

Kaya marapat lamang na paigtingin at paghusayin ang pagtuturo ng wika at panitikan upang muling manumbalik ang kawilihan sa pagbabasa at malinang ang kakayahan sa paggamit ng wika. Bilang pakikibahagi sa mithiing ito, isinagawa ang awtput hango sa kinalabasan ng isinagawang pag-aaral upang matugunan ang pangangailangan at maiangat ang pagtuturo ng wika at panitikan.

Kinatatampukan ang awtput na ito ng intergatibong banghay-aralin sa pagtuturo ng wika at panitikan. Ito ay isinakatuparan upang magabayan ang pagtuturo sa kasanayan sa wika at panitikan. Marami man ang hadlang na nagpapabagal sa pagpapayabong sa wika at panitikan hindi ito magiging balakid bagkus makatutulong ito upang hasain at pagtibayin pa ang kalinangan nito. Mahalaga rin ang awtput sapagkat naglalaman ito ng mga gawain na sadyang binalangkas upang mabigyan ng gabay ang pagtuturo ng wika at panitikan sa madali at simpleng paraan.

\section{Kongklusyon}

Nilalayon ng pag-aaral na ito na masuri ang mga piling maikling kwento ni P.S. Dandan bilang gabay sa pagbuo ng kagamitang pampagtuturo. Natuklasan sa banghay ng akdang "Mabangis na Kamay... Maamong Kamay", na nagsimula ito sa paglalarawan sa isang mahirap na bayan na pinalawig ng pagkakasakit ng anak ng pangunahing tauhan at winakasan sa pagkamatay nito. Sa banghay naman ng akdang "Lakas" sinimulan ito sa pagmumuni ng pangunahing tauhan na sinundan ng kanyang muling pagbangon laban sa pagiging paralitiko at winakasan sa isang realisasyon na nagpapakilos at gumagabay sa buhay. Sa akdang “Anino ng Kanyang Ama”, nagsimula ito sa isang tanong na pinalawig ng komprontasyon ng ama sa anak na winakasan sa kapighatian. Sa realismong pananaw naman ng banghay ng tatlong akda, nangingibabaw ang kahirapan ng buhay lalong-lalo na sa mga lugar na umaasa sa agrikultura samantalang ipinalulutang din nito ang suliranin sa kalusugan, panlalamang o pang-aabuso, pagmamaton ngunit ang mga suliraning ito ay hindi naging hadlang sa pananalig ng mga tauhan sa Dakilang Lumikha. Batay sa mga natuklasang ito, ipinalulutang lamang nito na ang banghay ng maikling kwento at ang mga isyung panlipunang umiiral sa mga akda ay humuhubog pa lalo sa kasiningan ng mga akda.

\subsection{Rekomendasyon}

Inirerekomenda na bigyang-diin ang banghay ng mga akda nang sa gayon ay madaling maunawaan at 
masundan ang daloy ng mga tagpo sa kwento.

$>\quad$ Himukin ang mga mag-aaral na tangkilikin ang mga maikling kwento at himayin ang realismong masisilayan sa mga akda upang mabuksan ang isipan ng mga mag-aaral sa usaping kinakaharap ng lipunan nang sa gayon ay maisasangkot nila ang sarili sa gawaing makabuluhan.

$>\quad$ Mainam din na gamitin ang awtput ng pag-aaral magsisilbing gabay sa pagtuturo ng wika at panitikan upang mahimok ang mga mag-aaral na pahalagahan ang mga akda at malinang pa lalo ang pag-unawa sa mga akdang pampanitikan.

Acknowledgement - Bahagi ng papel na ito ay naipresenta sa 1st National Conference for Arts, Sciences, and Education (N-CASE) na may temang “Accelerating Human Sciences Through Research" noong Pebrero 19-21, 2020 sa Waterfront Hotel, Cebu City, Philipines.

\section{Mga sanggunian}

Abad, R. G. (2001). Religion in the Philippines. Ateneo de Manila University, 49(3), 337-367.

Akap Bata Paty-List on Research and Education. (2012). Report on the condition of the Filipino youth [Ulat sa kalagayan ng batang Pilipino].

https://www.facebook.com/notes/akap-bata/ulat-sa-kalagayan-ng-batang-pilipino 2012/10151179124551067/

Balita. (2016). 50 percent of Filipinos feel poverty [50 porsyento ng mga pinoy ramdam ang kahirapan -SWS]. http://balita.net.ph/2016/01/07/50-porsyento-ng-mga-pinoy-ramdam-ang-kahirapansws/

CRI Online Filipino. (2015). Health, still a big issue in the Philippines [Kalusugan, isang malaking isyu pa rin sa Pilipinas]. http://filipino.cri.cn/301/2015/02/10/2s134543.htm

Gavilan, J. (2016). SWS: 10.5M Filipino families identify as poor in Q1 2016, http://www.rappler.com/nation/134464-sws-poverty-poll-first-quarter-2016

Lalic, E., \& Matic, A. (2004). Our Filipino literature [Ang Ating Panitikang Filipino]. Trinitas Publishing.

Oroc, J. E. (2012). Realism perspective on selected works of Patrocinio Villasan Villafuerte: Implication in teaching literature in tertiary [Realismong pananaw sa mga piling akda ni Patrocinio Villasan Villafuerte: implikasyon sa pagtuturo ng pampanitikang pantersyarya]. University of Visayas.

Perol, P. (2014). Cases of bullying in the Philippines [Kaso ng bullying sa Pilipinas], https://patriciaperol.wordpress.com/2016/10/14/kaso-ng-bullying-sa-pilipinas-2/

Philippine Social Scince Council. (2003). The Filipino youth: Some findings from research. https://books.google.com/books/about/The_Filipino_Youth.html?id=IDLaAAAAMAAJ

Philippine Statistics Office. (2014). Fishermen, farmers and children remain the poorest basic sectors. https://psa.gov.ph/content/fishermen-farmers-and-children-remain

Romualdez, Jr. A., dela Rosa J. F. E., Flavier, J. D., Quimbo, S. L. A., Hartigan-Go, K. Y., Lagrada L. P., \& David, L. C. (2011). The Phlippines health system review. World Health Organization, Regional Office for the Western Pacific.

Sauco, C. P., Papa, N. P., \& Geronimo, J. R. (2004). Literature of the Philippines (regional) [Panitikan ng Pilipinas (panrehiyon)]. Katha Publishing Co. Inc.

Villafuerte, P. V. (2000). Literary criticism (theory and exercise) [Panunuring Pampanitikan (teorya at pagsasanay)]. Mega-Jesta Prints.

Villafuerte, P. V., \& Bernales, R. A. (2008). Teaching Filipino: Theories and practices [Pagtuturo ng/sa Filipino: mga teorya at praktika]. Mutya Publishing House. 